Check for updates

Cite this: RSC Adv., 2018, 8, 34459

\title{
Tandem one-pot synthesis of 2-arylcinnolin-6-one derivatives from arylhydrazonopropanals and acetoacetanilides using sustainable ultrasound and microwave platforms $\dagger$
}

\author{
Hamad M. Al-Matar, (D) *a Kamal M. Dawood (D)*b and Wael M. Tohamy (D) \\ Several 2-arylcinnolin-6(2H)-one derivatives were synthesized via tandem annulation of a large number of \\ 3-oxo-2-arylhydrazonopropanals with acetoacetanilide under three different heating modes (conventional \\ heating, ultrasound and microwave irradiation) using triethylamine in ethanol. The factors affecting the \\ optimization of the annulation process were thoroughly studied. The annulated structures were \\ established on the basis of ${ }^{1} \mathrm{H}$ and ${ }^{13} \mathrm{C}$ NMR and MALDI-TOF/MS spectral data as well as single crystal X- \\ ray analysis.
}

Received 1st August 2018

Accepted 28th September 2018

DOI: $10.1039 / \mathrm{c} 8 \mathrm{ra0} 6494 \mathrm{f}$

rsc.li/rsc-advances

arylhydrazonopropanals ${ }^{23}$ diazotization of ortho-substituted

\section{Introduction}

Cinnolines are a class of aromatic nitrogen-heterocyclic compounds that are also known as benzo[c]pyridazines. Cinnoline derivatives have attracted considerable attention as drug candidates against several diseases due to their broad spectrum biological activities. ${ }^{1}$ They are patented as bactericides ${ }^{2-4}$ and fungicides. ${ }^{5}$ Cinnolin-3-one and cinnolin-4-one derivatives have also been reported to display potent antitumor, ${ }^{6-8}$ and antifungal, ${ }^{9}$ as well as human neutrophil elastase and STAT3 inhibitory activities ${ }^{10,11}$ and cannabinoid CB2 agonists. ${ }^{12,13}$ Cinnolin-5-ones were reported as atypical antipsychotic, ${ }^{14}$ and antitumor ${ }^{15}$ activities. In addition, cinnolin-3-carboxamides displayed ATM kinase inhibitors and were used in treating cancer ${ }^{16}$ and as BTK (Bruton's tyrosine kinase) inhibitors. ${ }^{17}$ Such potent biological importance of the cinnoline derivatives make them excellent templates for medicinal chemists to develop lead compounds for drug targets.

Thus, several reaction protocols have been reported for the synthesis of the cinnoline building blocks, among those protocols are the cyclization of the arylhydrazono-cyanoacetanilide, ${ }^{18}$ Richter cyclization of ortho-ethynyldiazonium salts, ${ }^{19-22}$ acid catalyzed cyclization of 3-oxo-3-aryl-2-

${ }^{a}$ Chemistry Department, Faculty of Science, University of Kuwait, P. O. Box 5969, Safat 13060, Kuwait. E-mail: h.almatar@ku.edu.kw; Fax: +965 24816482; Tel: +965 24987559

${ }^{b}$ Chemistry Department, Faculty of Science, Cairo University, Giza 12613, Egypt. E-mail:dr_dawood@yahoo.com; Fax:+20 235727556; Tel: +20 235676602

'Organometallic and Organometalloid Chemistry Department, National Research Centre, Cairo, Egypt

$\dagger$ Electronic supplementary information (ESI) available. CCDC 1858311. For ESI and crystallographic data in CIF or other electronic format see DOI: 10.1039/c8ra06494f anilines. ${ }^{24}$ The cinnolin-3(2H)-one derivatives were also synthesized by rhodium or gold catalyzed reactions of azobenzene derivatives. ${ }^{25,26}$ To date, the only reported cinnolinone ring skeletons included cinnolin-3-one, cinnolin-4-one, cinnolin-5-one, and cinnoline-5,6-dione derivatives, ${ }^{6-15,27,28}$ however, synthesis of cinnolin-6(2H)-one structures are not reported so far.

The environmentally sustainable ultrasound technique has pronounced applications in organic synthesis, medicinal chemistry, and in materials science. ${ }^{29-31}$ The ultrasound is reported to be superior over conventional heating in organic synthesis, where formation of pure products in high yields, high selectivity, shorter reaction time and waste-minimization are among the most advantages of ultrasound techniques. ${ }^{32-39}$ In addition, a growing interest is focused on the use of microwave irradiation methodology because it markedly assists in achieving rapid incorporation of organic synthesis into broad industrial diversities. ${ }^{40-43}$ In continuation of our research work employed ultrasound and microwave irradiations in heterocyclic synthesis, ${ }^{44-51}$ we envisaged in the current work a feasible simple and straightforward approach for the construction of 2-arylcinnolin-6(2H)-one derivatives, which would be assembled by reaction of 3-oxo-2arylhydrazonopropanals with double equivalents of acetoacetanilide via aldol-condensation followed by Michael-type addition reaction. For comparison with the conventional heating, two green and energy-saving platforms; ultrasound and microwave irradiation, are applied for achieving the goals of this work. Structures of the products are examined by measuring the X-ray crystallography along with all possible spectral analyses (IR, ${ }^{1} \mathrm{H}$ and ${ }^{13} \mathrm{C}$ NMR and MALDI-TOF/MS spectral data). 


\section{Results and discussion}

At first, 3-oxo-2-arylhydrazonopropanal derivatives 1a-o were synthesized using reported procedure in the literature..$^{5,53}$ Then, initial attempts to perform the annulation of the 3-oxo-2phenylhydrazonopropanal 1a with acetoacetanilide $\mathbf{2}$ in ethanol in the presence of triethylamine were conducted under conventional thermal heating. The expected pyridazine 4 or phenylazophenol 6 structures, according to the previously published protocols ${ }^{54,55}$ could not be detected, however the assigned product was established as 2-arylcinnolin-6(2H)-one derivative 3a (Scheme 1). To our delight, phenylhydrazonopropanal 1a satisfactorily reacted with double equivalent of acetoacetanilide 2 to give the aldol-condensation followed by Michael-type addition product 3a, through in situ elimination of three molecules of water during the reaction path (Scheme 1). The structure 3a was established on the basis of IR, ${ }^{1} \mathrm{H}$ and ${ }^{13} \mathrm{C}$ NMR spectra, MALDI-TOF/MS spectrum, and
X-ray diffraction data. Positive ion mode MALDI-TOF mass spectrum of 3a exhibited molecular ion peak of the form [M+ $\mathrm{K}]^{+}$at $m / z$ 624.317. Optimization of the reaction of $1 \mathrm{a}$ with 2 was thoroughly investigated under various reaction conditions (different solvents, bases and heating modes) and the reaction path was monitored by TLC till full conversion of the starting substrates, and the results are summarized in Table 1 . The results demonstrated that these factors greatly affected the productivity of this reaction. Among several solvents used (EtOH, MeOH, i-PrOH, $n$-hexane, DMF, 1,4-dioxane), ethanol was the proper one and the reaction was also much influenced by the type of base, where various organic and inorganic bases e.g. $\mathrm{Et}_{3} \mathrm{~N}$, pyridine, DBU, DABCO, $\mathrm{NaHCO}_{3}, \mathrm{~K}_{2} \mathrm{CO}_{3}$ and $\mathrm{KOH}$ were tested. Using ethanol as solvent and triethylamine (10 $\mathrm{mol} \%$ ) as base, the reaction of the hydrazonal 1a with 2 under conventional heating at reflux for 4 hours resulted in $66 \%$ isolated yield, however, $70 \%$ isolated yield was obtained after 7 min of ultrasound irradiation and the yield was $75 \%$ after only

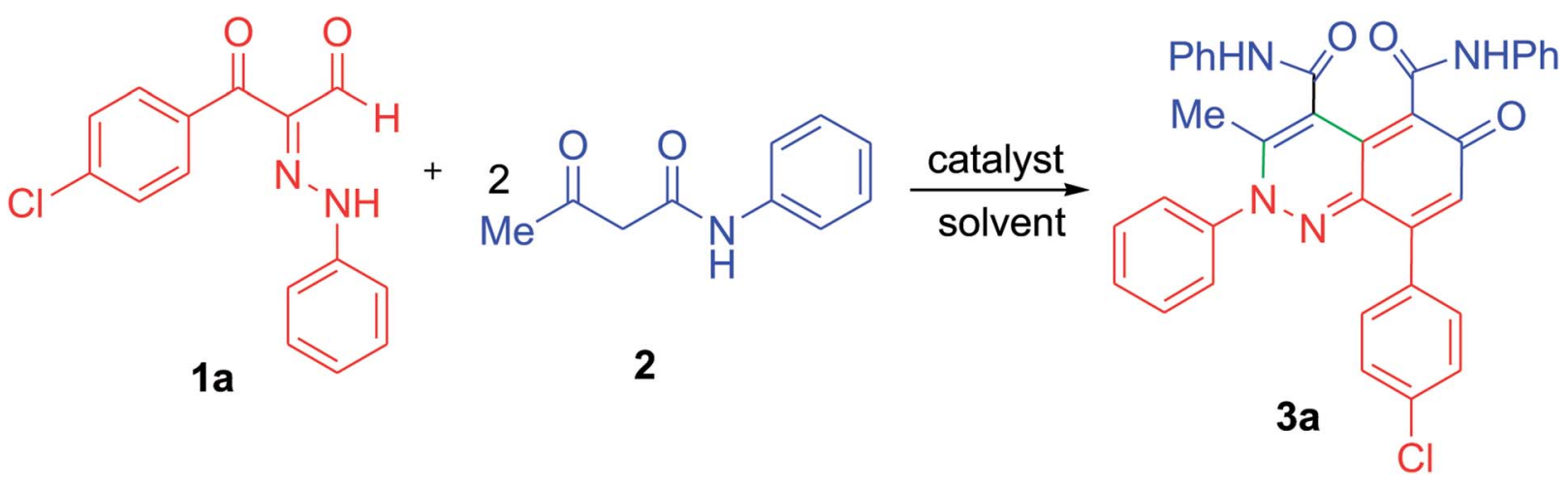

Scheme 1 Annulation of phenylhydrazonopropanal 1a with acetoacetanilide 2.

Table 1 Optimization the reaction condition of annulation of 1 a with $2^{a}$

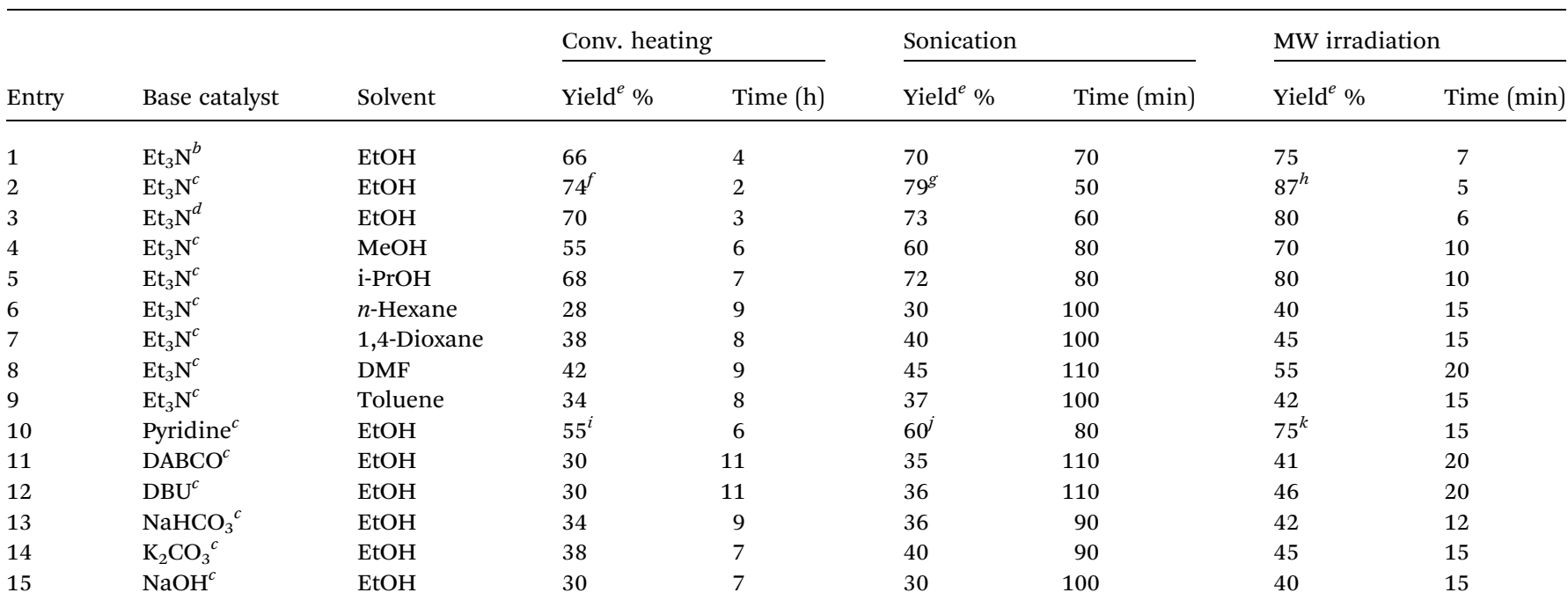

${ }^{a}$ Reaction conditions: phenylhydrazonopropanal 1a (2 mmol), acetoacetanilide 2 (4 mmol), solvent (15 mL) and base (10-30 mol\%), conventional heating at reflux for $2-11 \mathrm{~h}$, ultrasound at $80{ }^{\circ} \mathrm{C}$ for $50-110 \mathrm{~min}$, microwave irradiation at $80{ }^{\circ} \mathrm{C}(200 \mathrm{~W})$ for $5-20$ min. ${ }^{b}$ Base $10 \mathrm{~mol} \%{ }^{c}$ Base $20 \mathrm{~mol} \% .{ }^{d}$ Base $30 \mathrm{~mol} \% .{ }^{e}$ Isolated yields. ${ }^{f}$ Yield was $12 \%$ after $20 \mathrm{~min}$ and $50 \%$ after $80 \mathrm{~min} .{ }^{g}$ Yield was $30 \%$ after 20 min and $62 \%$ after 40 min. ${ }^{h}$ Yield was $53 \%$ after 3 min. ${ }^{i}$ Yield was $17 \%$ after 2 h. ${ }^{j}$ Yield was $38 \%$ after 50 min. ${ }^{k}$ Yield was $25 \%$ after 5 min. 
7 min of microwave irradiation (as examined by TLC) (entry 1 , Table 1). The use of $20 \mathrm{~mol} \%$ instead of $10 \mathrm{~mol} \%$ of triethylamine led to production of $74 \%, 79 \%$ and $87 \%$ isolated yields under conventional heating ( 2 hours), US irradiation (50 min) and MW irradiation (5 min), respectively (entry 2, Table 1). Minor shift to lower yields were observed when $\mathrm{Et}_{3} \mathrm{~N}$ was used in a higher ratio (30 mol\%), where the isolated yields became $70 \%$, $73 \%$ and $80 \%$ under thermal heating ( 3 hours), US (60 min) and MW (6 min), respectively (entry 3, Table 1). Very close product yields with low efficiency were obtained when $\mathrm{MeOH}$ or i-PrOH were employed as reaction solvent in the presence of $20 \mathrm{~mol} \%$ of $\mathrm{Et}_{3} \mathrm{~N}$ under all heating modes (entries 4 and 5, Table 1). Replacing ethanol with aprotic solvents (e.g. $n$-hexane, 1,4dioxane, DMF and toluene) led to a sharp decrease in the product yields (varied from 28-55\%) after longer reaction times regardless the type of heating mode (thermal, US or MW) (entries 6-9, Table 1). Keeping ethanol solvent and replacing $\mathrm{Et}_{3} \mathrm{~N}$ by pyridine resulted in $55 \%, 60 \%$ and $75 \%$ isolated yields under thermal heating (6 hours), US (80 min) and MW (15 min), respectively (entry 10, Table 1). Use of other bases such as DBU, DABCO, $\mathrm{NaHCO}_{3}, \mathrm{~K}_{2} \mathrm{CO}_{3}$ and $\mathrm{KOH}$ could not provide satisfactory yields (varied from $30-46 \%$ yields) under all the three heating modes (entries 11-15, Table 1). From the above findings, the benefit of microwave irradiation was confirmed by high efficiency with high conversion rate compared with the low conversion rate of the reactions that were carried out under conventional heating or ultrasound. The structure of the obtained reaction product 3a was unambiguously established by spectral analyses along with X-ray crystallography (Fig. 1). ${ }^{56}$ Mechanistically, polar solvents absorb microwave irradiation and convert it into heat that causes bulk heating and simultaneous rising of the temperature of the reaction mixture, in sharp contrast with conventional conductive heating. ${ }^{57}$ However, ultrasound waves cause cavitation phenomenon, that is, the creation, growth, and collapse of bubbles releasing enough energy to perform chemical reactions. ${ }^{31}$
Prompted by the above interesting results and under the optimized reaction conditions, generalization and scope of the reaction using different substrates were investigated as shown in Table 2. The procedure was found to be applicable to a wide range of the arylhydrazonopropanal derivatives 1a-o with acetoacetanilide 2 (Scheme 2). Thus, annulation reaction of arylhydrazonopropanals 1a-o with acetoacetanilide 2 was conducted using EtOH $(6 \mathrm{~mL})$ and $\mathrm{Et}_{3} \mathrm{~N}(20 \mathrm{~mol} \%)$ under the three heating modes; conventional heating, ultrasound irradiation and microwave irradiation, afforded the corresponding 2arylcinnolin-6(2H)-one derivatives 3a-o, Scheme 2 . The reaction was monitored by TLC till full conversions of the starting substrates and the isolated yields of the corresponding annulated products $3 \mathbf{3}-\mathbf{o}$ ranged from 45 to $75 \%$ (after $2-4$ hours under conventional heating), from 50 and 85\% yields (after 4080 min under ultrasound), and from 60 to $90 \%$ yields (after $3-$ 10 min of microwave irradiation) as depicted in Table 2, entries 1-15. From the structure-activity relationship point of view, it would be established, from the obtained results in Table 2, that the best yields within the shortest reaction times were obtained when $\mathrm{R}=4-\mathrm{FC}_{6} \mathrm{H}_{4}$ for the products $3 \mathrm{f}$ and $3 \mathrm{~g}$ among all the tested derivatives under all heating techniques (Table 2, entries 6 and 7). In contrast, the yields of the products $\mathbf{3 n}$ and $\mathbf{3 o}$ (where $\mathrm{R}=\mathrm{CH}_{3}$ ) were minimum and the reaction times were longest among all the derivatives under all applied heating modes (Table 2, entries 14 and 15). The 2-arylcinnolin-6(2H)-one structures 3a-o were determined from their all possible spectral data (IR, MALDI-TOF, ${ }^{1} \mathrm{H}$ and ${ }^{13} \mathrm{C}$ NMR spectra) and elemental analyses as mentioned in the Experimental section.

A plausible mechanistic pathway for the annulation of the hydrazonals 1a-o with acetoacetanilide 2 was suggested as depicted in Scheme 3. First, base catalyzed aldol condensation of the active methylene of 2 with the aldehyde function of 1 followed by loss of one molecule of water led to the formation of the $\alpha, \beta$-unsaturated carbonyl adduct $\mathbf{A}$. Then, a second molecule of $\mathbf{2}$ attacks the adduct $\mathbf{A}$ via a Michael-type addition to give

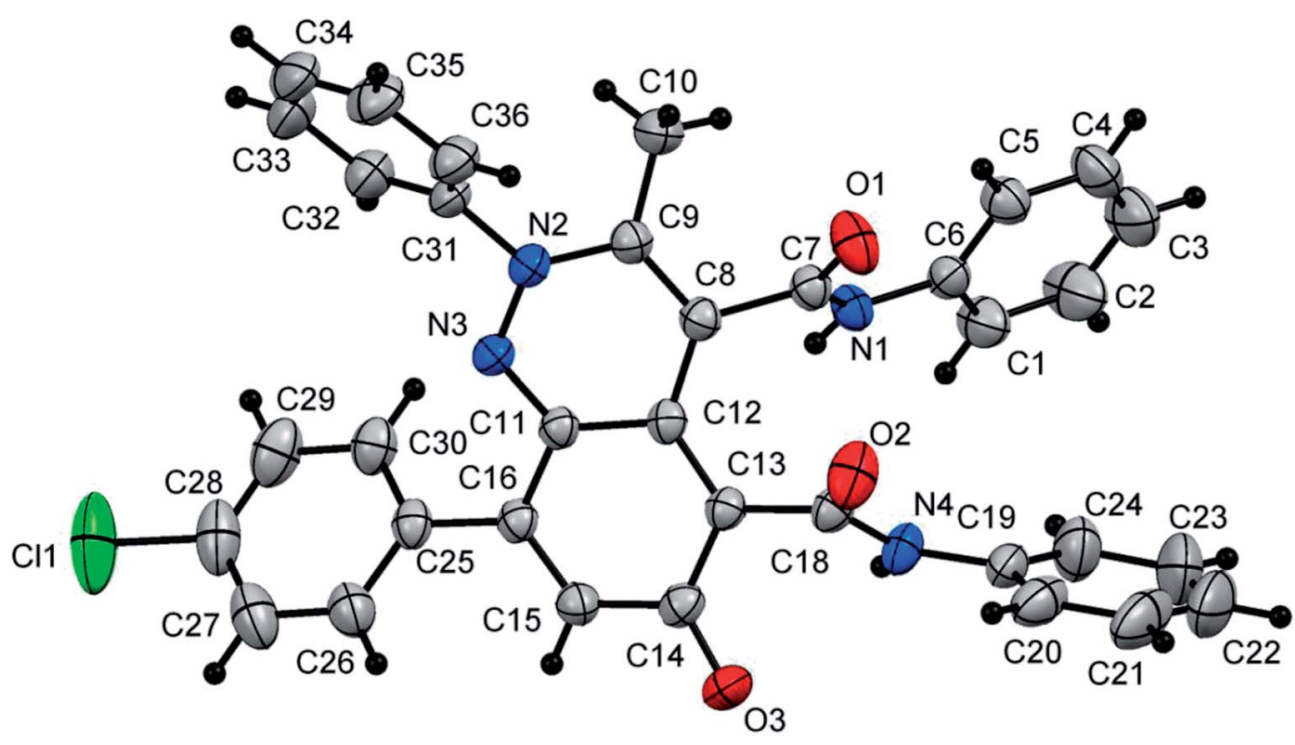

Fig. 1 ORTEP plot of the X-ray crystallographic data determined for 3a. 
Table 2 Tandem annulation of arylhydrazonopropanals 1a-o with acetoacetanilide 2 under conventional heating, ultrasound and microwave irradiations $^{a}$

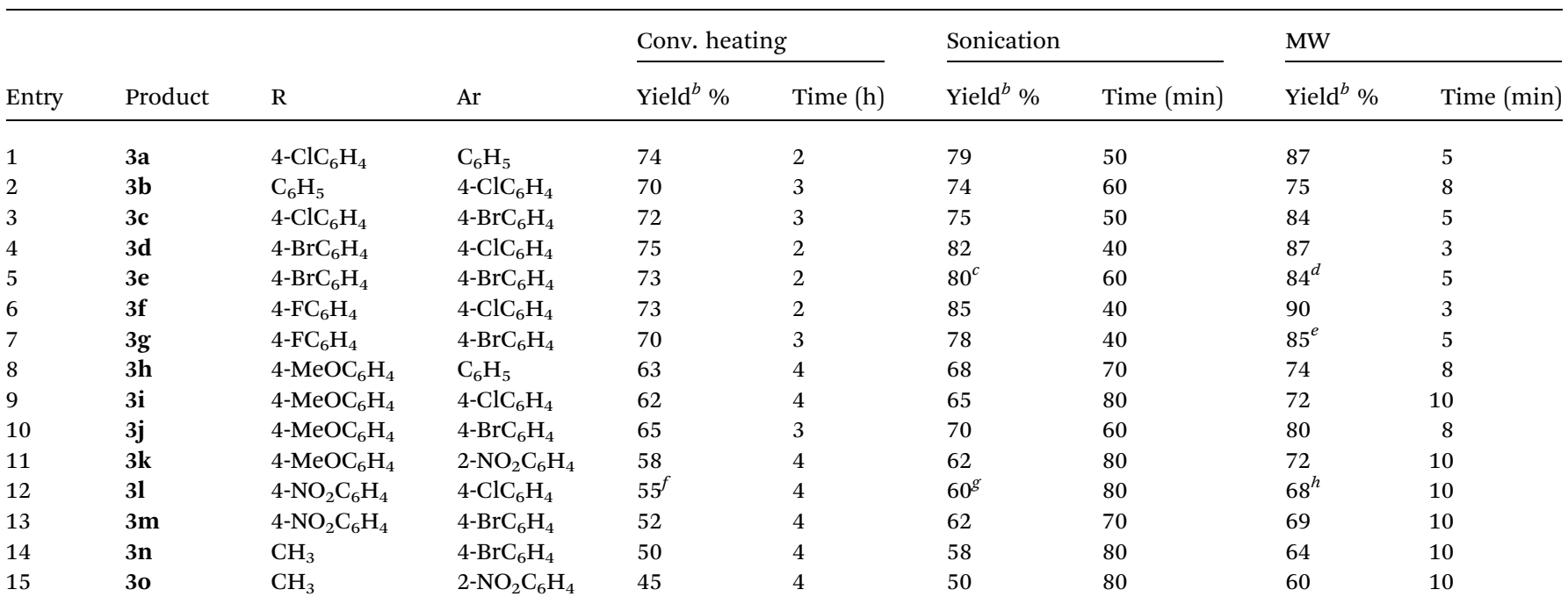

${ }^{a}$ Reaction condition: 1a-o $(1 \mathrm{mmol})$, acetoacetanilide $(2 \mathrm{mmol})$, triethylamine $(20 \mathrm{~mol} \%)$ in EtOH $(6 \mathrm{~mL})$, heated under reflux for $2-4 \mathrm{~h}$, ultrasound irradiation at $80{ }^{\circ} \mathrm{C}$ for $40-80 \mathrm{~min}$, microwave irradiations at $80{ }^{\circ} \mathrm{C}(200 \mathrm{~W})$ for $3-10 \mathrm{~min} .{ }^{b}$ Isolated yields. ${ }^{c}$ Yield was $55 \%$ after 40 min. ${ }^{d}$ Yield was $51 \%$ after 3 min. ${ }^{e}$ Yield was $52 \%$ after 3 min. ${ }^{f}$ Yield was $28 \%$ after 2 h. ${ }^{g}$ Yield was $31 \%$ after 40 min. ${ }^{h}$ Yield was $21 \%$ after 3 min.

the intermediate $\mathbf{B}$, which underwent cyclization via nucleophilic attack of the hydrazone- $\mathrm{NH}$ to $\mathrm{C}=\mathrm{O}$ function followed by loss of water molecule from $\mathbf{C}$ to give the pyridazine intermediate D. Further annulations took place by base catalyzed cyclization through $\mathrm{C}-\mathrm{C}$ bond formation followed by loss of water molecule from $\mathbf{E}$ to give the intermediate $\mathbf{F}$. Loss of hydrogen molecule through air oxidation of $\mathbf{F}$ led to the formation of the novel 6-oxo-2,6-dihydrocinnoline-4,5dicarboxamide derivatives $\mathbf{3 a - o}$.

\section{Experimental section}

\section{General}

Melting points were recorded on a Griffin melting point apparatus and are reported uncorrected. IR spectra were recorded using KBr disks using a Perkin-Elmer System 2000 FT-IR spectrophotometer. ${ }^{1} \mathrm{H}-\mathrm{NMR}(600 \mathrm{MHz})$ and ${ }^{13} \mathrm{C}-\mathrm{NMR}(150 \mathrm{MHz})$ spectra were recorded at $25{ }^{\circ} \mathrm{C}$ using DMSO- $\mathrm{d}_{6}$ as solvent with TMS as internal standard on a Bruker DPX 400 or 600 superconducting NMR spectrometer. Chemical shifts are reported in ppm. Low-resolution electron impact mass spectra [MS (EI)] was performed using a high resolution GC-MS (DFS) thermo spectrometer at $70.1 \mathrm{eV}$ using magnetic sector mass analyzer. Follow up of the reactions was made by using thin layer chromatography (TLC). Microwave experiments were carried out using a CEM Discover LabMate ${ }^{\circledR}$ microwave apparatus $(300 \mathrm{~W}$ with ChemDriver software; Matthews, NC). Reactions were conducted under microwave irradiation in heavy-walled Pyrex tubes fitted with PCS caps (closed vessel under pressure). The Xray crystal structures were determined by using a Rigaku R-AXIS RAPID diffractometer and Bruker X8 Prospector and the collection of single crystal data was made at room temperature by using $\mathrm{Cu}-\mathrm{K} \alpha$ radiation. The data were collected at room temperature. The structures were solved by using direct methods and expanded using Fourier techniques. The nonhydrogen atoms were refined anisotropically. The structures were solved and refined using the Bruker SHELXTL Software Package (Structure solution program-SHELXS-97 and Refinement program-SHELXL 97).$^{58}$ Data were corrected for the absorption effects using the multi-scan method (SADABS). Sonication was performed in MKC6, Guyson ultrasonic bath (Model-MKC6, operating frequency $38 \mathrm{kHz} \pm 10 \%$ and an<smiles>[R]C(=O)/C(=N/N[Ga]O[Na])C(=O)[18O]c1ccccc1</smiles><smiles>[R]c1cc(=O)c(C(N)=O)c2c(C(=O)Nc3ccccc3)c(C)n([Ga])nc1-2</smiles>

Scheme 2 Annulation of arylhydrazonopropanals 1a-o with acetoacetanilide 2 . 


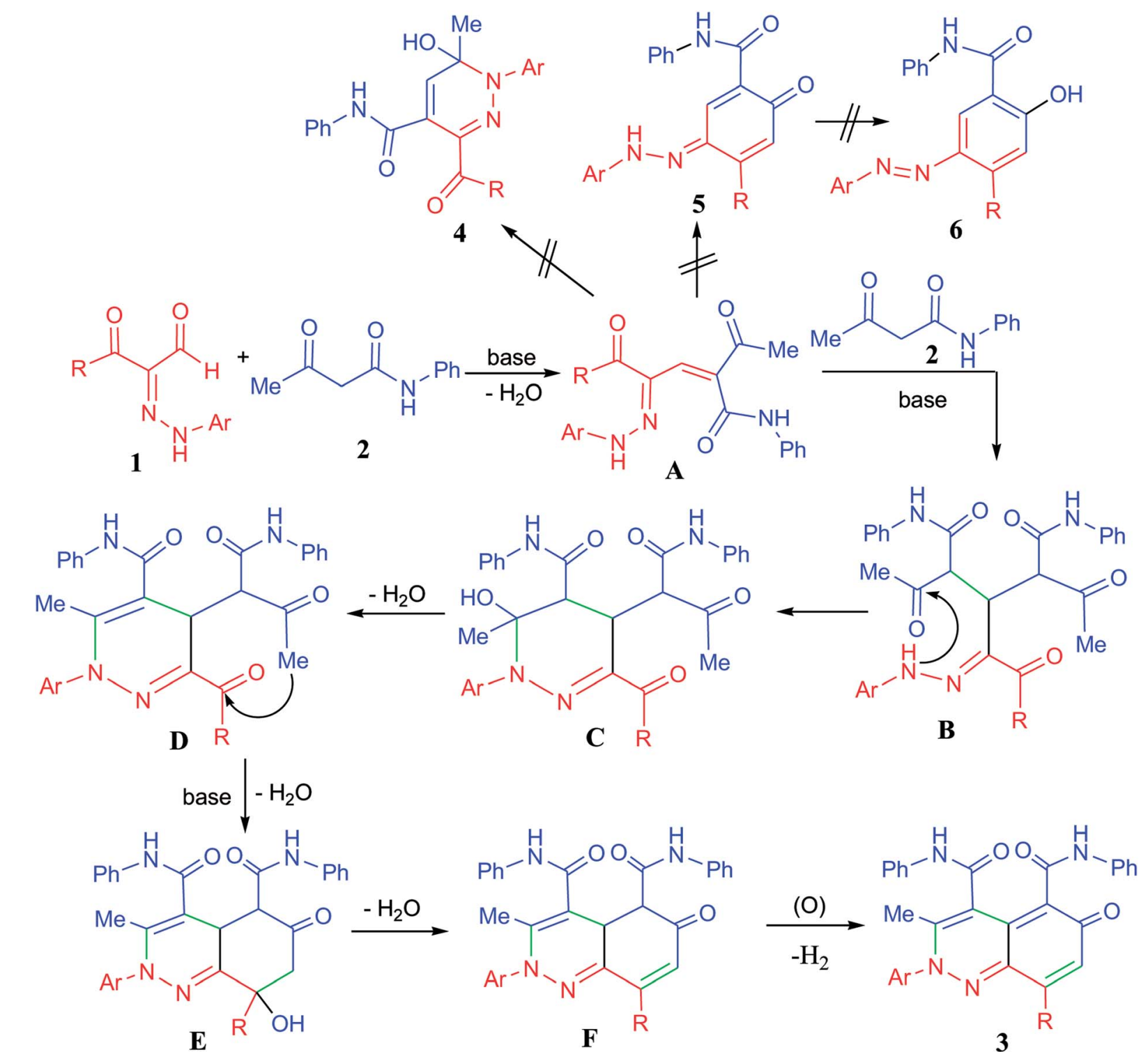

Scheme 3 Proposed mechanism for the annulation pathway.

output power of 110 watts) with digital timer (6 s to $100 \mathrm{~min}$ ) and heater allows solution heating to be set from 20 to $80{ }^{\circ} \mathrm{C}$ in $1{ }^{\circ} \mathrm{C}$ increments. The inside tank dimensions are $150 \times 300 \times$ $150 \mathrm{~mm}$ (length $\times$ width $\times$ depth) with a fluid capacity of $6 \mathrm{~L}$.

\section{Molecular weight determination using MALDI-TOF/MS}

Determination of molecular weight was achieved using MALDITOF/MS (Bruker, model ultraflexXtreme). All the analyses were examined in positive mode using a reflectron time of flight mass spectrometer. Data processing was performed using Bruker flexAnalysis. The instrument was calibrated with peptide calibration standard.

The sample was prepared as following: the matrix $\alpha$-cyano-4hydroxycinnamic acid (HCCA) was purchased from Sigma Aldrich. All reagents were used without any further purification. HCCA was dissolved in mixture of acetonitrile : water : trifluoroacetic acid $(70: 30: 0.1)$. All samples were dissolved in chloroform. Deposition of the samples on target pate was done as follows: $5 \mathrm{~L}$ of the sample was mixed with $5 \mathrm{~L}$ of the matrix (1:1 ratio). The solution mixture was placed on the target plate and allowed to dry at room temperature.

\section{Synthesis of 6-oxo-2,6-dihydrocinnoline-4,5-dicarboxamide derivatives $3 a-0$}

General method A. To a mixture of the appropriate arylhydrazonopropanal 1a-o $(2 \mathrm{mmol})$ and acetoacetanilide $2(0.71 \mathrm{~g}$, $4 \mathrm{mmol}$ ) in the appropriate solvent (EtOH, MeOH, i-PrOH, $n$ hexane, DMF or 1,4-dioxane) $(15 \mathrm{~mL})$, triethylamine $(0.06 \mathrm{~mL}$, $20 \mathrm{~mol} \%$ ) was added portion-wise. The mixture was heated at refluxing temperature till the starting substrates were almost completely consumed as followed up by TLC, then left to cool to room temperature. The solvent was removed under reduced pressure to give yellow or orange colored solid products. The solid products were recrystallized from dimethylformamide 
(DMF)/ethanol to afford the corresponding 6-oxo-cinnoline-4,5dicarboxamide derivatives $\mathbf{3 a - 0}$ as pure products.

General method B. A mixture of the appropriate arylhydrazonopropanal 1a-o ( $2 \mathrm{mmol})$ and acetoacetanilide $2(0.71 \mathrm{~g}$, $4 \mathrm{mmol})$ and triethylamine $(0.06 \mathrm{~mL}, 20 \mathrm{~mol} \%)$ in the appropriate solvent (EtOH, MeOH, i-PrOH, $n$-hexane, DMF or 1,4dioxane) (15 mL) was mixed thoroughly. The mixture was sonicated in a MKC6, Guyson ultrasonic bath (Model-MKC6, operating frequency $38 \mathrm{kHz} \pm 10 \%$ and an output power of $110 \mathrm{~W}$ ) for $40-80 \mathrm{~min}$ at $80{ }^{\circ} \mathrm{C}$ during which time the starting substrates were almost completely consumed as followed up by TLC, then left to cool to room temperature. The solvent was removed under reduced pressure to give yellow or orange colored solid products. The solid products were recrystallized from dimethylformamide (DMF)/ethanol to afford the corresponding 6-oxo-cinnoline-4,5-dicarboxamide derivatives 3a-o as pure products.

General method C. A mixture of the appropriate arylhydrazonopropanal 1a-o $(2 \mathrm{mmol})$ and acetoacetanilide $2(0.71 \mathrm{~g}$, $4 \mathrm{mmol})$ and triethylamine $(0.06 \mathrm{~mL}, 20 \mathrm{~mol} \%)$ in the appropriate solvent (EtOH, MeOH, i-PrOH, $n$-hexane, DMF or 1,4dioxane) (15 mL) was mixed thoroughly in a process glass vial. The vial was capped properly, and thereafter, the mixture was heated under microwave irradiating conditions at $80{ }^{\circ} \mathrm{C}$ and $200 \mathrm{~W}$ for the appropriate reaction time as listed in Table 2. Then mixture was then left to cool to room temperature and the solvent was removed under reduced pressure to give yellow or orange colored solid products. The solid products were recrystallized from dimethylformamide (DMF)/ethanol to afford the corresponding 6-oxo-cinnoline-4,5-dicarboxamide derivatives 3a-o as pure products.

8-(4-Chlorophenyl)-3-methyl-6-oxo- $N^{4}, N^{5}, 2$-triphenyl-2, 6dihydrocinnoline-4,5-dicarboxamide (3a). Orange crystals, $\operatorname{mp} 268-270{ }^{\circ} \mathrm{C}$; IR (KBr): $\nu / \mathrm{cm}^{-1} 3259(\mathrm{NH}), 1683,1654(\mathrm{C}=\mathrm{O})$; ${ }^{1} \mathrm{H}$ NMR (DMSO-d $): \delta=2.35\left(\mathrm{~s}, 3 \mathrm{H}, \mathrm{CH}_{3}\right), 6.95(\mathrm{t}, J=6.9 \mathrm{~Hz}, 1 \mathrm{H}$, $\mathrm{Ar}-\mathrm{H}), 7.06(\mathrm{t}, J=6.6 \mathrm{~Hz}, 1 \mathrm{H}, \mathrm{Ar}-\mathrm{H}), 7.11(\mathrm{~m}, 3 \mathrm{H}, \mathrm{Ar}-\mathrm{H}), 7.24(\mathrm{t}, J$ $=7.8 \mathrm{~Hz}, 2 \mathrm{H}, \mathrm{Ar}-\mathrm{H}), 7.46-7.47(\mathrm{~m}, 2 \mathrm{H}, \mathrm{Ar}-\mathrm{H}), 7.49-7.50(\mathrm{~m}, 2 \mathrm{H}$, $\mathrm{Ar}-\mathrm{H}), 7.55-7.62(\mathrm{~m}, 3 \mathrm{H}, \mathrm{Ar}-\mathrm{H}), 7.64$ (d, $J=4.2 \mathrm{~Hz}, 4 \mathrm{H}, \mathrm{Ar}-\mathrm{H})$, 7.66-7.68 (m, 2H, Ar-H), 10.48 (s, 1H, NH), 10.55 (s, 1H, NH); ${ }^{13} \mathrm{C}$ NMR $\left(\mathrm{DMSO}-\mathrm{d}_{6}\right): \delta=19.35\left(\mathrm{CH}_{3}\right), 115.17,119.48,119.79$, 123.50, 124.20, 125.94, 127.33, 127.97, 128.09, 128.36, 129.78, 131.90, 133.46, 134.43, 135.41, 138.74, 139.43, 141.73, 142.56 (Ar-C) 162.59, 164.20, $178.96(\mathrm{C}=\mathrm{O})$. MALDI-TOF: calcd for $[\mathrm{M}+$ $\mathrm{K}]^{+} m / z=624.128$, found $m / z=624.317$. Anal. calcd for $\mathrm{C}_{35} \mathrm{H}_{25} \mathrm{ClN}_{4} \mathrm{O}_{3}$ : C, 71.85; H, 4.31; N, 9.58 found: C, 71.77; H, 4.39; N, 9.61. Crystal data, $\mathrm{C}_{35} \mathrm{H}_{25} \mathrm{ClN}_{4} \mathrm{O}_{3}, M=585.04$, monoclinic, $a=15.5696(6) \AA, b=19.6736(7) \AA, c=9.4182(3) \AA, \alpha=$ $90^{\circ}, \beta=95.241(3)^{\circ}, \gamma=90^{\circ}, V=2872.83(18) \AA^{3}, T=296(2) \mathrm{K}$, space group: $P 121 / c 1, Z=4$, calculated density $=1.353 \mathrm{~g} \mathrm{~cm}^{-3}$, no. of reflection measured $24182, \theta_{\max }=66.51^{\circ}, R 1=0.0800 .^{56}$

2-(4-Chlorophenyl)-3-methyl-6-oxo- $N^{4}, N^{5}, 8$-triphenyl-2,6dihydrocinnoline-4,5-dicarboxamide (3b). Yellow crystals, $\mathrm{mp}$ 270-271 ${ }^{\circ} \mathrm{C}$; IR (KBr): $\nu / \mathrm{cm}^{-1} 3249(\mathrm{NH}), 1677,1660(\mathrm{C}=\mathrm{O}) ;{ }^{1} \mathrm{H}$ NMR (DMSO-d $\left.\mathrm{d}_{6}\right): \delta=2.36\left(\mathrm{~s}, 3 \mathrm{H}, \mathrm{CH}_{3}\right) ; 6.97(\mathrm{t}, J=7.2 \mathrm{~Hz}, 1 \mathrm{H}$, $\mathrm{Ar}-\mathrm{H}), 7.07-7.15$ (m, 2H, Ar-H), 7.13 (t, $J=7.8 \mathrm{~Hz}, 2 \mathrm{H}, \mathrm{Ar}-\mathrm{H})$, $7.25(\mathrm{t}, J=8.1 \mathrm{~Hz}, 2 \mathrm{H}, \mathrm{Ar}-\mathrm{H}), 7.42-7.49(\mathrm{~m}, 5 \mathrm{H}, \mathrm{Ar}-\mathrm{H}), 7.56(\mathrm{~d}, J$ $=7.8 \mathrm{~Hz}, 2 \mathrm{H}, \mathrm{Ar}-\mathrm{H}), 7.65(\mathrm{~d}, J=8.4 \mathrm{~Hz}, 2 \mathrm{H}, \mathrm{Ar}-\mathrm{H}), 7.63-7.72(\mathrm{~m}$,
$4 \mathrm{H}, \mathrm{Ar}-\mathrm{H}), 10.48(\mathrm{~s}, 1 \mathrm{H}, \mathrm{NH}), 10.56(\mathrm{~s}, 1 \mathrm{H}, \mathrm{NH}) ;{ }^{13} \mathrm{C}$ NMR $\left(\mathrm{DMSO}-\mathrm{d}_{6}\right): \delta=19.33\left(\mathrm{CH}_{3}\right), 115.41,119.49,119.79,122.54$, 123.53 , 123.95, 127.23, 127.92, 127.98, 128.11, 128.39, 128.59, 129.79, 130.11, 134.49, 135.23, 135.58, 138.75, 139.47, 140.59, 141.32, 141.59, 143.32 (Ar-C), 162.55, 164.23, $178.80(\mathrm{C}=\mathrm{O})$. MALDI-TOF: calcd for $[\mathrm{M}+\mathrm{Na}]^{+} m / z=607.151,[\mathrm{M}+\mathrm{K}]^{+} m / z=$ 623.128, found $m / z=607.50$ and 623.458; anal. calcd for $\mathrm{C}_{35} \mathrm{H}_{25} \mathrm{ClN}_{4} \mathrm{O}_{3}$ : C, 71.85; H, 4.31; N, 9.58. Found: C, 71.71; H, $4.40 ; \mathrm{N}, 9.51$.

2-(4-Bromophenyl)-8-(4-chlorophenyl)-3-methyl-6-oxo- $N^{4}, N^{5}$ diphenyl-2,6-dihydrocinnoline-4,5-dicarboxamide (3c). Pale yellow crystals, mp 274-276 ${ }^{\circ} \mathrm{C}$; IR (KBr): $\nu / \mathrm{cm}^{-1} 3251(\mathrm{NH})$,

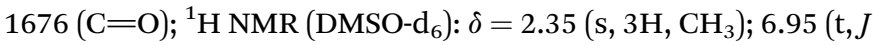
$=7.25 \mathrm{~Hz}, 1 \mathrm{H}, \mathrm{Ar}-\mathrm{H}), 7.06(\mathrm{t}, J=7.2 \mathrm{~Hz}, 1 \mathrm{H}, \mathrm{Ar}-\mathrm{H}), 7.10-7.13$ $(\mathrm{m}, 3 \mathrm{H}, \mathrm{Ar}-\mathrm{H}), 7.24(\mathrm{t}, J=7.8 \mathrm{~Hz}, 2 \mathrm{H}, \mathrm{Ar}-\mathrm{H}), 7.45(\mathrm{~d}, J=7.2 \mathrm{~Hz}$, $2 \mathrm{H}, \mathrm{Ar}-\mathrm{H}), 7.50$ (d, $J=8.4 \mathrm{~Hz}, 2 \mathrm{H}, \mathrm{Ar}-\mathrm{H}), 7.53$ (d, $J=7.8 \mathrm{~Hz}, 2 \mathrm{H}$, $\mathrm{Ar}-\mathrm{H}), 7.61$ (d, $J=8.4 \mathrm{~Hz}, 2 \mathrm{H}, \mathrm{Ar}-\mathrm{H}), 7.67$ (d, $J=8.4 \mathrm{~Hz}, 2 \mathrm{H}, \mathrm{Ar}-$ $\mathrm{H}), 7.85(\mathrm{~d}, J=8.4 \mathrm{~Hz}, 2 \mathrm{H}, \mathrm{Ar}-\mathrm{H}), 10.44(\mathrm{~s}, 1 \mathrm{H}, \mathrm{NH}), 10.55$ (s, 1H, $\mathrm{NH}) ;{ }^{13} \mathrm{C}$ NMR (DMSO-d $): \delta=19.39\left(\mathrm{CH}_{3}\right), 115.56,119.52$, 119.82, 122.59, 123.56, 123.16, 123.58, 124.04, 127.22, 128.03, $128.13,128.15,128.41,131.94,132.80,133.55,134.36,135.39$, 138.74, 139.45, 140.40, 141.63, 141.73, 142.03 (Ar-C), 162.55, 164.18, $178.68(\mathrm{C}=\mathrm{O})$. MALDI-TOF: calcd for $[\mathrm{M}+\mathrm{K}]^{+} \mathrm{m} / z=$ 703.034, found 703.713; anal. calcd for $\mathrm{C}_{35} \mathrm{H}_{24} \mathrm{BrClN}_{4} \mathrm{O}_{3}: \mathrm{C}$, 63.31; H, 3.64; N, 8.44. Found: C, 63.28; H, 3.72; N, 8.49.

8-(4-Bromophenyl)-2-(4-chlorophenyl)-3-methyl-6-oxo- $N^{4}, N^{5}$ diphenyl-2,6-dihydrocinnoline-4,5-dicarboxamide (3d). Orange crystals, mp 276-278 ${ }^{\circ} \mathrm{C}$; IR (KBr): $\nu / \mathrm{cm}^{-1} 3247(\mathrm{NH}), 1674(\mathrm{C}=$ O); ${ }^{1} \mathrm{H}$ NMR (DMSO-d $\left.{ }_{6}\right): \delta=2.34\left(\mathrm{~s}, 3 \mathrm{H}, \mathrm{CH}_{3}\right), 6.95(\mathrm{t}, J=7.2 \mathrm{~Hz}$, $1 \mathrm{H}, \mathrm{Ar}-\mathrm{H}), 7.06$ (t, J=7.2 Hz, 1H, Ar-H), $7.11(\mathrm{~m}, 3 \mathrm{H}, \mathrm{Ar}-\mathrm{H}), 7.24$ $(\mathrm{t}, J=8.1 \mathrm{~Hz}, 2 \mathrm{H}, \mathrm{Ar}-\mathrm{H}), 7.46(\mathrm{~d}, J=7.8 \mathrm{~Hz}, 2 \mathrm{H}, \mathrm{Ar}-\mathrm{H}), 7.54(\mathrm{~d}, J$ $=7.8 \mathrm{~Hz}, 2 \mathrm{H}, \mathrm{Ar}-\mathrm{H}), 7.60-7.65(\mathrm{~m}, 4 \mathrm{H}, \mathrm{Ar}-\mathrm{H}), 7.67-7.73(\mathrm{~m}, 4 \mathrm{H}$, $\mathrm{Ar}-\mathrm{H}), 10.44$ (s, 1H, NH), 10.54 (s, 1H, NH); ${ }^{13} \mathrm{C}$ NMR (DMSO-d 6 ): $\delta=19.37\left(\mathrm{CH}_{3}\right), 115.54,119.49,119.79,122.24,122.56,123.55$, $124.00,127.22,128.11,129.85,130.94,132.21,134.55,134.73$, 135.36, 138.73, 139.44, 140.31, 141.29, 141.67, 142.07 (Ar-C), 162.53, 164.15, $179.43(\mathrm{C}=\mathrm{O})$. MALDI-TOF: calcd for $[\mathrm{M}+\mathrm{Na}]^{+}$ $m / z=686.937,[\mathbf{M}+\mathbf{K}]^{+} m / z=703.045$, found $m / z=687.294$ and 703.261; anal. calcd For $\mathrm{C}_{35} \mathrm{H}_{24} \mathrm{BrClN}_{4} \mathrm{O}_{3}$ : C, 63.31; H, 3.64; N, 8.44. Found: C, 63.38; H, 3.60; N, 8.41.

2,8-Di(4-bromophenyl)-3-methyl-6-oxo- $N^{4}, N^{5}$-diphenyl-2,6dihydrocinnoline-4,5-dicarboxamide (3e). Pale yellow solid, mp 268-270 ${ }^{\circ} \mathrm{C}$; IR (KBr): $\nu / \mathrm{cm}^{-1} 3250(\mathrm{NH}), 1676(\mathrm{C}=\mathrm{O}) ;{ }^{1} \mathrm{H}$ NMR (DMSO-d $\left.\mathrm{d}_{6}\right): \delta=2.34\left(\mathrm{~s}, 3 \mathrm{H}, \mathrm{CH}_{3}\right), 6.95(\mathrm{t}, J=7.5 \mathrm{~Hz}, 1 \mathrm{H}$, $\mathrm{Ar}-\mathrm{H}), 7.06$ (t, $J=7.5 \mathrm{~Hz}, 1 \mathrm{H}, \mathrm{Ar}-\mathrm{H}), 7.10-7.13$ (m, 3H, Ar-H), $7.24(\mathrm{t}, J=7.8 \mathrm{~Hz}, 2 \mathrm{H}, \mathrm{Ar}-\mathrm{H}), 7.46(\mathrm{~d}, J=7.8 \mathrm{~Hz}, 2 \mathrm{H}, \mathrm{Ar}-\mathrm{H}), 7.54$ (d, $J=7.8 \mathrm{~Hz}, 2 \mathrm{H}, \mathrm{Ar}-\mathrm{H}), 7.59-7.65(\mathrm{~m}, 6 \mathrm{H}, \mathrm{Ar}-\mathrm{H}), 7.86$ (d, $J=$ $9 \mathrm{~Hz}, 2 \mathrm{H}, \mathrm{Ar}-\mathrm{H}), 10.44$ (s, 1H, NH), 10.54 (s, 1H, NH); ${ }^{13} \mathrm{C}$ NMR $\left(\mathrm{DMSO}-\mathrm{d}_{6}\right): \delta=19.37\left(\mathrm{CH}_{3}\right), 115.55,119.49,119.79,122.24$, $122.56,123.15,123.55,124.01,127.20,128.11,128.14,128.39$, $130.94,132.20,132.79,134.72,135.35,138.72,139.44,140.31$, 141.60, 141.71, 142.05 (Ar-C), 162.52, 164.15, $178.65(\mathrm{C}=\mathrm{O})$. MALDI-TOF: calcd for $[\mathrm{M}+\mathrm{Na}]^{+} \mathrm{m} / z=729.011$, found 729.03; anal. calcd for $\mathrm{C}_{35} \mathrm{H}_{24} \mathrm{Br}_{2} \mathrm{~N}_{4} \mathrm{O}_{3}$ : C, 59.34; H, 3.41; N, 7.91. Found: C, 59.22; H, 3.49; N, 7.84.

2-(4-Chlorophenyl)-8-(4-fluorophenyl)-3-methyl-6-oxo- $N^{4}, N^{5}$ diphenyl-2,6-dihydrocinnoline-4,5-dicarboxamide (3f). Yellow 
solid, mp 271-273 ${ }^{\circ} \mathrm{C}$; IR (KBr): $\nu / \mathrm{cm}^{-1} 3277(\mathrm{NH}), 1659(\mathrm{C}=\mathrm{O})$; ${ }^{1} \mathrm{H}$ NMR (DMSO-d $\left.{ }_{6}\right): \delta=2.35\left(\mathrm{~s}, 3 \mathrm{H}, \mathrm{CH}_{3}\right), 6.95(\mathrm{t}, J=7.5 \mathrm{~Hz}, 1 \mathrm{H}$, $\mathrm{Ar}-\mathrm{H}), 7.06$ (t, $J=7.2 \mathrm{~Hz}, 1 \mathrm{H}, \mathrm{Ar}-\mathrm{H}), 7.09(\mathrm{~s}, 1 \mathrm{H}, \mathrm{Ar}-\mathrm{H}), 7.12(\mathrm{t}, J$ $=7.8 \mathrm{~Hz}, 2 \mathrm{H}, \mathrm{Ar}-\mathrm{H}), 7.22-7.29(\mathrm{~m}, 4 \mathrm{H}, \mathrm{Ar}-\mathrm{H}), 7.45(\mathrm{~d}, J=7.2 \mathrm{~Hz}$, $2 \mathrm{H}, \mathrm{Ar}-\mathrm{H}), 7.55$ (d, $J=7.2 \mathrm{~Hz}, 2 \mathrm{H}, \mathrm{Ar}-\mathrm{H}), 7.67-7.72(\mathrm{~m}, 6 \mathrm{H}, \mathrm{Ar}-$ H), 10.46 (s, 1H, NH), 10.55 (s, 1H, NH); ${ }^{13} \mathrm{C}$ NMR (DMSO-d ${ }_{6}$ ): $\delta=19.38\left(\mathrm{CH}_{3}\right), 114.86,115.00,115.44,119.51,119.80,122.57$, 123.56, 124.01, 127.25, 127.92, 128.12, 128.41, 129.83, 131.87, 131.89, 132.26, 132.31, 134.51, 135.26, 138.75, 139.46, 140.54, 141.33, 141.61, 142.20 (Ar-C), 162.59, 164.20, $178.75(\mathrm{C}=\mathrm{O})$. MALDI-TOF: calcd for $[\mathrm{M}+\mathrm{Na}]^{+} \mathrm{m} / \mathrm{z}=626.031$, found 626.317; anal. calcd for $\mathrm{C}_{35} \mathrm{H}_{24} \mathrm{ClFN}_{4} \mathrm{O}_{3}: \mathrm{C}, 69.71 ; \mathrm{H}, 4.01 ; \mathrm{N}, 9.29$. Found: C, 69.65; H, 4.15; N, 9.23.

2-(4-Bromophenyl)-8-(4-fluorophenyl)-3-methyl-6-oxo- $N^{4}, N^{5}$ diphenyl-2,6-dihydrocinnoline-4,5-dicarboxamide (3g). Yellow crystals, mp $267-269^{\circ} \mathrm{C}$; IR (KBr): $\nu / \mathrm{cm}^{-1} 3274(\mathrm{NH}), 1676(\mathrm{C}=$ O); ${ }^{1} \mathrm{H}$ NMR (DMSO-d $\left.\mathrm{d}_{6}\right): \delta=2.35\left(\mathrm{~s}, 3 \mathrm{H}, \mathrm{CH}_{3}\right) ; 6.95(\mathrm{t}, J=7.2 \mathrm{~Hz}$, $1 \mathrm{H}, \mathrm{Ar}-\mathrm{H}), 7.06(\mathrm{t}, J=7.5 \mathrm{~Hz}, 1 \mathrm{H}, \mathrm{Ar}-\mathrm{H}), 7.09(\mathrm{~s}, 1 \mathrm{H}, \mathrm{Ar}-\mathrm{H}), 7.12$ $(\mathrm{t}, J=7.8 \mathrm{~Hz}, 2 \mathrm{H}, \mathrm{Ar}-\mathrm{H}), 7.22-7.29(\mathrm{~m}, 4 \mathrm{H}, \mathrm{Ar}-\mathrm{H}), 7.47$ (d, $J=$ $7.2 \mathrm{~Hz}, 2 \mathrm{H}, \mathrm{Ar}-\mathrm{H}), 7.55$ (d, $J=7.2 \mathrm{~Hz}, 2 \mathrm{H}, \mathrm{Ar}-\mathrm{H}), 7.61$ (d, $J=$ $8.4 \mathrm{~Hz}, 2 \mathrm{H}, \mathrm{Ar}-\mathrm{H}), 7.68-7.71(\mathrm{~m}, 2 \mathrm{H}, \mathrm{Ar}-\mathrm{H}), 7.85(\mathrm{~d}, J=9 \mathrm{~Hz}, 2 \mathrm{H}$, $\mathrm{Ar}-\mathrm{H}$ ), 10.46 (s, 1H, NH), 10.57 (s, 1H, NH); ${ }^{13} \mathrm{C}$ NMR (DMSO-d 6 ): $\delta=19.38\left(\mathrm{CH}_{3}\right), 114.85,114.99,115.45,119.50,122.55,123.10$, 123.53, 124.02, 127.24, 128.11, 128.14, 128.39, 131.87, 132.24, 132.29, 132.76, 135.25, 138.75, 139.45, 140.54, 141.54, 141.74, 142.19, 161.54, 162.27 (Ar-C) 163.17, 164.19, 178.75 (C=O). MALDI-TOF: calcd for [M $+\mathrm{Na}]^{+} \mathrm{m} / z=669.091$, found 669.316; anal. calcd for $\mathrm{C}_{35} \mathrm{H}_{24} \mathrm{BrFN}_{4} \mathrm{O}_{3}: \mathrm{C}, 64.92 ; \mathrm{H}, 3.74 ; \mathrm{N}, 8.65$. Found: C, 64.85; H, 3.66; N, 8.77.

8-(4-Anisyl)-3-methyl-6-oxo- $\mathrm{N}^{4}, \mathrm{~N}^{5}, 2$-triphenyl-2, 6-dihydrocinnoline4,5-dicarboxamide (3h). Orange crystals, $\mathrm{mp} 242-244{ }^{\circ} \mathrm{C} ; \mathrm{IR}(\mathrm{KBr})$ : $\nu / \mathrm{cm}^{-1} 3260(\mathrm{NH}), 1687,1653(\mathrm{C}=\mathrm{O}) ;{ }^{1} \mathrm{H}$ NMR $\left(\right.$ DMSO-d $\left._{6}\right): \delta=$ $2.35\left(\mathrm{~s}, 3 \mathrm{H}, \mathrm{CH}_{3}\right), 3.79\left(\mathrm{~s}, 3 \mathrm{H}, \mathrm{OCH}_{3}\right), 6.96(\mathrm{t}, J=7.5 \mathrm{~Hz}, 1 \mathrm{H}, \mathrm{Ar}-$ H), $7.00(\mathrm{~d}, J=9 \mathrm{~Hz}, 1 \mathrm{H}, \mathrm{Ar}-\mathrm{H}), 7.07-7.14(\mathrm{~m}, 4 \mathrm{H}, \mathrm{Ar}-\mathrm{H}), 7.24(\mathrm{t}$, $J=7.8 \mathrm{~Hz}, 2 \mathrm{H}, \mathrm{Ar}-\mathrm{H}), 7.38-7.48(\mathrm{~m}, 4 \mathrm{H}, \mathrm{Ar}-\mathrm{H}), 7.57-7.61(\mathrm{~m}$, $4 \mathrm{H}, \mathrm{Ar}-\mathrm{H}), 7.64-7.82(\mathrm{~m}, 3 \mathrm{H}, \mathrm{Ar}-\mathrm{H}), 7.95(\mathrm{~d}, J=7.8 \mathrm{~Hz}, 2 \mathrm{H}, \mathrm{Ar}-$ H), 11.84 (br. s, 2H, 2NH); ${ }^{13} \mathrm{C}$ NMR (DMSO-d 6 ): $\delta=19.45\left(\mathrm{CH}_{3}\right)$, $55.77\left(\mathrm{OCH}_{3}\right), 113.43,113.56,114.24,114.91,115.37,116.61$, 119.57, 119.87, 122.59, 124.18, 125.83, 126.07, 127.90, 128.46, 129.85, 131.57, 132.42, 138.89, 139.29, 141.63, 142.70 (Ar-C), 162.67, 164.42, $188.79(\mathrm{C}=\mathrm{O})$. MALDI-TOF: calcd for $[\mathrm{M}+\mathrm{Na}]^{+}$ $m / z=603.201$, found 603.327; anal. calcd for $\mathrm{C}_{36} \mathrm{H}_{28} \mathrm{~N}_{4} \mathrm{O}_{4}$ : C, 74.47; H, 4.86; N, 9.65. Found: C, 74.40; H, 4.74; N, 9.77.

8-(4-Anisyl)-2-(4-chlorophenyl)-3-methyl-6-oxo- $N^{4}, N^{5}$-diphenyl2,6-dihydrocinnoline-4,5-dicarboxamide (3i). Orange crystals, mp 225-227 ${ }^{\circ} \mathrm{C}$; IR (KBr): $\nu / \mathrm{cm}^{-1} 3313(\mathrm{NH}), 1705,1642(\mathrm{C}=\mathrm{O})$; ${ }^{1} \mathrm{H}$ NMR (DMSO-d $\left.\mathrm{d}_{6}\right): \delta=2.34\left(\mathrm{~s}, 3 \mathrm{H}, \mathrm{CH}_{3}\right), 3.79\left(\mathrm{~s}, 3 \mathrm{H}, \mathrm{OCH}_{3}\right)$, $6.95(\mathrm{t}, J=7.8 \mathrm{~Hz}, 1 \mathrm{H}, \mathrm{Ar}-\mathrm{H}), 7.00(\mathrm{~d}, J=8.4 \mathrm{~Hz}, 2 \mathrm{H}, \mathrm{Ar}-\mathrm{H}), 7.04$ (s, 1H, Ar-H), 7.07 (d, $J=7.2 \mathrm{~Hz}, 1 \mathrm{H}, \mathrm{Ar}-\mathrm{H}), 7.12(\mathrm{t}, J=7.8 \mathrm{~Hz}$, $2 \mathrm{H}, \mathrm{Ar}-\mathrm{H}), 7.24(\mathrm{t}, J=7.8 \mathrm{~Hz}, 2 \mathrm{H}, \mathrm{Ar}-\mathrm{H}), 7.47(\mathrm{~d}, J=7.2 \mathrm{~Hz}, 2 \mathrm{H}$, $\mathrm{Ar}-\mathrm{H}), 7.56(\mathrm{~d}, J=7.8 \mathrm{~Hz}, 2 \mathrm{H}, \mathrm{Ar}-\mathrm{H}), 7.60(\mathrm{~d}, J=8.4 \mathrm{~Hz}, 2 \mathrm{H}, \mathrm{Ar}-$ $\mathrm{H})$, 7.68-7.73 (m, 4H, Ar-H), $10.50(\mathrm{~s}, 1 \mathrm{H}, \mathrm{NH}), 10.53(\mathrm{~s}, 1 \mathrm{H}$, $\mathrm{NH}) ;{ }^{13} \mathrm{C}$ NMR (DMSO-d $\left.)_{6}\right): \delta=19.35\left(\mathrm{CH}_{3}\right), 55.18\left(\mathrm{OCH}_{3}\right)$, 113.50, 115.18, 119.49, 119.79, 122.53, 123.51, 123.91, 127.34, 127.73, 127.93, 128.12, 128.39, 129.82, 131.50, 134.45, 138.78, 139.46, 140.68, 141.36, 141.46, 142.93, 159.70 (Ar-C), 162.66, 164.28, $178.95(\mathrm{C}=\mathrm{O})$. MALDI-TOF: calcd for $[\mathrm{M}+\mathrm{Na}]^{+} \mathrm{m} / \mathrm{z}=$
637.162, $[\mathrm{M}+\mathrm{K}]^{+} m / z=653.136$, found 637.488 and 653.447; anal. calcd For $\mathrm{C}_{36} \mathrm{H}_{27} \mathrm{ClN}_{4} \mathrm{O}_{4}$ : C, 70.30; H, 4.42; N, 9.11. Found: C, 70.40; H, 4.55; N, 9.18.

8-(4-Anisyl)-2-(4-bromophenyl)-3-methyl-6-oxo- $N^{4}, N^{5}$-diphenyl-2,6dihydrocinnoline-4,5-dicarboxamide (3j). Orange crystals, mp 252$254^{\circ} \mathrm{C}$; IR (KBr): $\nu / \mathrm{cm}^{-1} 3268(\mathrm{NH}), 1678,1659(\mathrm{C}=\mathrm{O}) ;{ }^{1} \mathrm{H}$ NMR (DMSO-d $\left.\mathrm{d}_{6}\right): \delta=2.34\left(\mathrm{~s}, 3 \mathrm{H}, \mathrm{CH}_{3}\right), 3.79\left(\mathrm{~s}, 3 \mathrm{H}, \mathrm{OCH}_{3}\right), 6.97(\mathrm{t}, J=$ $7.5 \mathrm{~Hz}, 1 \mathrm{H}, \mathrm{Ar}-\mathrm{H}), 7.02(\mathrm{~d}, J=8.4 \mathrm{~Hz}, 2 \mathrm{H}, \mathrm{Ar}-\mathrm{H}), 7.07(\mathrm{t}, J=$ $7.5 \mathrm{~Hz}, 2 \mathrm{H}, \mathrm{Ar}-\mathrm{H}), 7.12(\mathrm{t}, J=7.8 \mathrm{~Hz}, 2 \mathrm{H}, \mathrm{Ar}-\mathrm{H}), 7.24(\mathrm{t}, J=$ $7.8 \mathrm{~Hz}, 2 \mathrm{H}, \mathrm{Ar}-\mathrm{H}), 7.46(\mathrm{~d}, J=7.8 \mathrm{~Hz}, 2 \mathrm{H}, \mathrm{Ar}-\mathrm{H}), 7.55-7.62(\mathrm{~m}$, $6 \mathrm{H}, \mathrm{Ar}-\mathrm{H}), 7.86(\mathrm{~d}, J=8.4 \mathrm{~Hz}, 2 \mathrm{H}, \mathrm{Ar}-\mathrm{H}), 10.49(\mathrm{~s}, 1 \mathrm{H}, \mathrm{NH})$, $10.54(\mathrm{~s}, 1 \mathrm{H}, \mathrm{NH}) ;{ }^{13} \mathrm{C}$ NMR $\left(\right.$ DMSO-d $\left._{6}\right): \delta=19.39\left(\mathrm{CH}_{3}\right), 55.20$ $\left(\mathrm{OCH}_{3}\right), 113.52$, 115.24, 119.51, 119.80, 122.56, 123.08, 123.54, 123.92, 127.32, 127.74, 128.14, 128.19, 128.42, 131.52, 132.78, 134.41, 138.80, 139.49, 140.70, 141.42, 141.80, 142.95, 159.72 (Ar-C), 162.68, 164.30, $178.96(\mathrm{C}=\mathrm{O})$. MALDI-TOF: calcd for [M $+\mathrm{H}]^{+} m / z=661.127$, found 661.456; anal. calcd for $\mathrm{C}_{36} \mathrm{H}_{27} \mathrm{BrN}_{4} \mathrm{O}_{4}$ : C, 65.56; $\mathrm{H}, 4.13 ; \mathrm{N}, 8.49$. Found: C, 65.40; $\mathrm{H}$, $4.23 ; \mathrm{N}, 8.58$.

8-(4-Anisyl)-3-methyl-2-(4-nitrophenyl)-6-oxo- $N^{4}, N^{5}$-diphenyl2,6-dihydrocinnoline-4,5-dicarboxamide (3k). Orange crystals, $\mathrm{mp} 248-250{ }^{\circ} \mathrm{C}$; IR (KBr): $\nu / \mathrm{cm}^{-1} 3274(\mathrm{NH}), 1661(\mathrm{C}=\mathrm{O}) ;{ }^{1} \mathrm{H}$ NMR (DMSO-d $\left.{ }_{6}\right): \delta=2.35\left(\mathrm{~s}, 3 \mathrm{H}, \mathrm{CH}_{3}\right), 3.78\left(\mathrm{~s}, 3 \mathrm{H}, \mathrm{OCH}_{3}\right), 6.92-$ $7.02(\mathrm{~m}, 5 \mathrm{H}, \mathrm{Ar}-\mathrm{H}), 7.13(\mathrm{t}, J=7.8 \mathrm{~Hz}, 2 \mathrm{H}, \mathrm{Ar}-\mathrm{H}), 7.22-7.31(\mathrm{~m}$, $4 \mathrm{H}, \mathrm{Ar}-\mathrm{H}), 7.47(\mathrm{~m}, 2 \mathrm{H}, \mathrm{Ar}-\mathrm{H}), 7.54-7.58(\mathrm{~m}, 3 \mathrm{H}, \mathrm{Ar}-\mathrm{H}), 7.89(\mathrm{t}, J$ $=7.8 \mathrm{~Hz}, 1 \mathrm{H}, \mathrm{Ar}-\mathrm{H}), 8.01(\mathrm{~s}, 1 \mathrm{H}, \mathrm{Ar}-\mathrm{H}), 8.36(\mathrm{~d}, J=7.8 \mathrm{~Hz}, 1 \mathrm{H}$, Ar-H), 10.40 (br. s, 1H, NH), 10.64 (s, 1H, NH); ${ }^{13} \mathrm{C}$ NMR (DMSO$\left.\mathrm{d}_{6}\right): \delta=19.39\left(\mathrm{CH}_{3}\right), 55.19\left(\mathrm{OCH}_{3}\right), 113.40,113.61,119.43$, 119.52, 119.78, 119.93, 122.46, 122.69, 123.59, 123.54, 126.68, 127.54, 128.07, 128.10, 128.32, 128.41, 128.73, 130.91, 131.52, 131.94, 134.53, 134.85, 135.37, 138.63, 139.54, 141.43, 141.95, $142.95,144.05, \quad 159.58,159.64 \quad(\mathrm{Ar}-\mathrm{C}), 162.20,164.64$, 179.15(C=O). MALDI-TOF: calcd for $[\mathrm{M}+\mathrm{Na}]^{+} \mathrm{m} / \mathrm{z}=648.186$, found 648.439; anal. calcd for $\mathrm{C}_{36} \mathrm{H}_{27} \mathrm{~N}_{5} \mathrm{O}_{6}: \mathrm{C}, 69.11 ; \mathrm{H}, 4.35 ; \mathrm{N}$, 11.19. Found: C, 69.22; $\mathrm{H}, 4.42 ; \mathrm{N}, 11.26$.

2-(4-Chlorophenyl)-3-methyl-8-(4-nitrophenyl)-6-oxo- $N^{4}, N^{5}$ diphenyl-2,6-dihydrocinnoline-4,5-dicarboxamide (3l). Orange crystals, mp $249-251^{\circ} \mathrm{C}$; IR (KBr): $\nu / \mathrm{cm}^{-1} 3251(\mathrm{NH}), 1668(\mathrm{C}=$ O); ${ }^{1} \mathrm{H}$ NMR (DMSO-d $\left.\mathrm{d}_{6}\right): \delta=2.35\left(\mathrm{~s}, 3 \mathrm{H}, \mathrm{CH}_{3}\right), 6.95(\mathrm{t}, J=7.5 \mathrm{~Hz}$, $1 \mathrm{H}, \mathrm{Ar}-\mathrm{H}), 7.07(\mathrm{t}, J=7.5 \mathrm{~Hz}, 1 \mathrm{H}, \mathrm{Ar}-\mathrm{H}), 7.12(\mathrm{t}, J=7.5 \mathrm{~Hz}, 2 \mathrm{H}$, $\mathrm{Ar}-\mathrm{H}), 7.22-7.25(\mathrm{~m}, 3 \mathrm{H}, \mathrm{Ar}-\mathrm{H}), 7.47$ (d, $J=7.8 \mathrm{~Hz}, 2 \mathrm{H}, \mathrm{Ar}-\mathrm{H})$, $7.55(\mathrm{~d}, J=7.8 \mathrm{~Hz}, 2 \mathrm{H}, \mathrm{Ar}-\mathrm{H}), 7.68-7.72(\mathrm{~m}, 4 \mathrm{H}, \mathrm{Ar}-\mathrm{H}), 7.94(\mathrm{~d}, J$ $=8.4 \mathrm{~Hz}, 2 \mathrm{H}, \mathrm{Ar}-\mathrm{H}), 8.26(\mathrm{~d}, J=8.4 \mathrm{~Hz}, 2 \mathrm{H}, \mathrm{Ar}-\mathrm{H}), 10.41(\mathrm{~s}, 1 \mathrm{H}$, $\mathrm{NH}), 10.56(\mathrm{~s}, 1 \mathrm{H}, \mathrm{NH}) ;{ }^{13} \mathrm{C}$ NMR $\left(\mathrm{DMSO}_{\mathrm{C}}\right)$ : $\delta=19.87\left(\mathrm{CH}_{3}\right)$, 116.44, 119.99, 120.30, 123.09, 123.47, 124.09, 124.59, 127.61, 128.40, 128.61, 128.89, 130.37, 131.98, 135.07, 136.70, 139.18, 139.92, 140.66, 141.72, 142.35, 142.64, 147.86 (Ar-C), 162.92, 164.55, $178.84(\mathrm{C}=\mathrm{O})$. MALDI-TOF: calcd for $[\mathrm{M}+\mathrm{Na}]^{+} \mathrm{m} / \mathrm{z}=$ 652.136, found 652.859; anal. calcd for $\mathrm{C}_{35} \mathrm{H}_{24} \mathrm{ClN}_{5} \mathrm{O}_{5}$ : C, 66.72; $\mathrm{H}, 3.84 ; \mathrm{N}, 11.12$. Found: C, 66.66; H, 3.95; N, 11.29.

2-(4-Bromophenyl)-3-methyl-8-(4-nitrophenyl)-6-oxo- $N^{4}, N^{5}$ diphenyl-2,6-dihydrocinnoline-4,5-dicarboxamide (3m). Orange solid, mp 302-304 ${ }^{\circ} \mathrm{C}$; IR (KBr): $\nu / \mathrm{cm}^{-1} 3259(\mathrm{NH}), 1668(\mathrm{C}=\mathrm{O})$; ${ }^{1} \mathrm{H}$ NMR (DMSO-d ${ }_{6}$ ): $\delta=2.36\left(\mathrm{~s}, 3 \mathrm{H}, \mathrm{CH}_{3}\right), 6.96-7.26(\mathrm{~m}, 7 \mathrm{H}, \mathrm{Ar}-$ $\mathrm{H}), 7.47(\mathrm{~d}, J=7.2 \mathrm{~Hz}, 2 \mathrm{H}, \mathrm{Ar}-\mathrm{H}), 7.56$ (d, $J=7.8 \mathrm{~Hz}, 2 \mathrm{H}, \mathrm{Ar}-\mathrm{H})$, 7.60-7.65 (m, 2H, Ar-H), 7.86-7.95 (m, 4H, Ar-H), 8.27 (d, $J=$ $7.8 \mathrm{~Hz}, 1 \mathrm{H}, \mathrm{Ar}-\mathrm{H}), 8.34$ (d, J=7.8 Hz, 1H, Ar-H), 10.45 (s, 1H, 
$\mathrm{NH}), 10.57$ (s, 1H, NH); ${ }^{13} \mathrm{C}$ NMR (DMSO-d 6$): \delta=19.39\left(\mathrm{CH}_{3}\right)$, $115.70,115.81,119.52$, 119.82, 121.76, 122.60, 122.98, 123.16, 123.21, 123.60, 124.03, 124.69, 127.20, 128.12, 128.40, 130.78, 131.08, 131.48, 132.81, 135.92, 136.85, 138.74, 139.44, 140.35, 140.45, 141.66, 142.28, 143.46, 147.41 (Ar-C), 162.28, 164.18, $178.68(\mathrm{C}=\mathrm{O})$. MALDI-TOF: calcd for $[\mathrm{M}+\mathrm{Na}]^{+} \mathrm{m} / z=698.038$, found 698.295; anal. calcd For $\mathrm{C}_{35} \mathrm{H}_{24} \mathrm{BrN}_{5} \mathrm{O}_{5}$ : C, 62.32; $\mathrm{H}$, 3.59; N, 10.38. Found: C, 62.26; H, 3.46; N, 10.42 .

2-(4-Bromophenyl)-3,8-dimethyl-6-oxo- $N^{4}, N^{5}$-diphenyl-2,6dihydrocinnoline-4,5-dicarboxamide (3n). Orange crystals, $\mathrm{mp}$ 222-223 ${ }^{\circ} \mathrm{C}$; IR (KBr): $\nu / \mathrm{cm}^{-1} 3240(\mathrm{NH}), 1658,1640(\mathrm{C}=\mathrm{O}) ;{ }^{1} \mathrm{H}$ NMR (DMSO-d $\left.\mathrm{d}_{6}\right): \delta=2.34\left(\mathrm{~s}, 3 \mathrm{H}, \mathrm{CH}_{3}\right), 2.39\left(\mathrm{~s}, 3 \mathrm{H}, \mathrm{CH}_{3}\right), 6.95(\mathrm{t}$, $J=6.9 \mathrm{~Hz}, 1 \mathrm{H}, \mathrm{Ar}-\mathrm{H}), 6.99(\mathrm{~s}, 1 \mathrm{H}, \mathrm{Ar}-\mathrm{H}), 7.06(\mathrm{t}, J=7.5 \mathrm{~Hz}, 1 \mathrm{H}$, $\mathrm{Ar}-\mathrm{H}), 7.12(\mathrm{t}, J=7.5 \mathrm{~Hz}, 2 \mathrm{H}, \mathrm{Ar}-\mathrm{H}), 7.23(\mathrm{t}, J=7.8 \mathrm{~Hz}, 2 \mathrm{H}, \mathrm{Ar}-$ $\mathrm{H}), 7.45$ (d, $J=7.2 \mathrm{~Hz}, 2 \mathrm{H}, \mathrm{Ar}-\mathrm{H}), 7.55$ (d, $J=7.2 \mathrm{~Hz}, 2 \mathrm{H}, \mathrm{Ar}-\mathrm{H})$, 7.66 (d, $J=7.8 \mathrm{~Hz}, 2 \mathrm{H}, \mathrm{Ar}-\mathrm{H}), 7.91$ (d, $J=7.8 \mathrm{~Hz}, 2 \mathrm{H}, \mathrm{Ar}-\mathrm{H})$, 10.43 (s, 1H, NH), 10.47 (s, 1H, NH); ${ }^{13} \mathrm{C}$ NMR (DMSO-d ${ }_{6}$ ): $\delta=$ $16.88\left(\mathrm{CH}_{3}\right), 19.27\left(\mathrm{CH}_{3}\right), 114.32,119.49,119.77,122.50,123.16$, 123.49, 123.61, 127.44, 128.11, 128.38, 132.86, 135.14, 138.80, 139.47, 140.68, 141.58, 141.92, 142.27 (Ar-C), 162.66, 164.30, $179.51(\mathrm{C}=\mathrm{O})$. MALDI-TOF: calcd for $[\mathrm{M}+\mathrm{Na}]^{+} \mathrm{m} / z=589.085$, found 589.513; anal. calcd For $\mathrm{C}_{30} \mathrm{H}_{23} \mathrm{BrN}_{4} \mathrm{O}_{3}$ : C, 63.50; $\mathrm{H}$, 4.09; N, 9.87. Found: C, 63.55; H, 4.14; N, 9.76.

3,8-Dimethyl-2-(2-nitrophenyl)-6-oxo- $N^{4}, N^{5}$-diphenyl-2, 6dihydrocinnoline-4,5-dicarboxamide (3o). Orange crystals, mp 176-178 ${ }^{\circ} \mathrm{C}$; IR (KBr): $\nu / \mathrm{cm}^{-1} 3260(\mathrm{NH}), 1677(\mathrm{C}=\mathrm{O}) ;{ }^{1} \mathrm{H}$ NMR (DMSO-d $\left.\mathrm{d}_{6}\right): \delta=2.37\left(\mathrm{~s}, 3 \mathrm{H}, \mathrm{CH}_{3}\right), 2.38\left(\mathrm{~s}, 3 \mathrm{H}, \mathrm{CH}_{3}\right), 7.06(\mathrm{t}$, $J=7.2 \mathrm{~Hz}, 2 \mathrm{H}, \mathrm{Ar}-\mathrm{H}), 7.11-7.14(\mathrm{~m}, 2 \mathrm{H}, \mathrm{Ar}-\mathrm{H}), 7.31(\mathrm{t}, J=$ $8.1 \mathrm{~Hz}, 2 \mathrm{H}, \mathrm{Ar}-\mathrm{H}), 7.57(\mathrm{~d}, J=8.4 \mathrm{~Hz}, 2 \mathrm{H}, \mathrm{Ar}-\mathrm{H}), 7.73-7.75(\mathrm{~m}$, 2H, Ar-H), 7.83 (s, 2H, Ar-H), 7.92-7.94 (m, 2H, Ar-H), 8.12 (d, $J$ $=8.4 \mathrm{~Hz}, 2 \mathrm{H}, \mathrm{Ar}-\mathrm{H}), 11.52(\mathrm{~s}, 2 \mathrm{H}, 2 \mathrm{NH}) ;{ }^{13} \mathrm{C}$ NMR $\left(\mathrm{DMSO}_{6}\right)$ : $\delta=24.50\left(\mathrm{CH}_{3}\right), 30.17\left(\mathrm{CH}_{3}\right), 115.02,116.84,119.06,119.50$, 120.96, 121.22, 123.39, 125.70, 128.74, 130.02, 133.55, 135.22, 136.12, 138.87, 139.15, 141.13 (Ar-C) 165.02, 197.16, 202.83 (C= O). MALDI-TOF: calcd for $[\mathrm{M}+\mathrm{Na}]^{+} \mathrm{m} / z=556.159$, found 556.233; anal. calcd For $\mathrm{C}_{30} \mathrm{H}_{23} \mathrm{~N}_{5} \mathrm{O}_{5}$ : C, 67.53; H, 4.35; N, 13.13. Found: C, 67.59; H, 4.28; N, 13.24.

\section{Conclusions}

A feasible and efficient one-pot tandem annulation of the hydrazonals 1a-o with acetoacetanilide 2 was performed under three different heating modes (conventional heating, ultrasound and microwave irradiation) and resulted in the formation of a series of novel class of the 2-arylcinnolin-6(2H)-one derivatives 3a-o. Microwave irradiation proved to be superior and efficient tool over ultrasound and conventional heating, for the promotion of such annulation reactions using ethanol as solvent and $\mathrm{Et}_{3} \mathrm{~N}$ as a base.

\section{Conflicts of interest}

There are no conflicts to declare.

\section{Acknowledgements}

This research work was financially supported by the University of Kuwait through a research grant (SC07/13). The facilities of ANALAB and SAF supported by research grants GS01/01, GS01/ 05, GS01/03, and GS03/08 are greatly appreciated.

\section{References}

1 W. Lewgowd and A. Stanczak, Arch. Pharm. Chem. Life Sci., 2007, 340, 65-80.

2 S. Inoue, A. Yasaki, H. Mochizuki, H. Tutsumi, M. Murata and K. Sakane, Jpn. Pat., 05213951 A2 930824, 1993, Chem. Abstr., 1993, 120, 134503w.

3 H. Tutsumi, T. Terasawa, D. Barret, M. Murata, K. Sakane, A. Yazaki and S. Inoue, Jpn. Pat., 9215584 A1 920217, 1992, Chem. Abstr., 1992, 118, 254944w.

4 M. Yokomoto, A. Yazaki, N. Hayashi, S. Hatono, S. Ioue and Y. Kuramoto, Eur. Pat., 4700578 A1 920212, 1992, Chem. Abstr., 1992, 117, 7943c.

5 M. J. Coghlan, B. A. Driekorn, R. G. Suhr and G. P. Jourdan, Eur. Pat., 0326328 A2 890802, 1989, Chem. Abstr., 1989, 112, 55907 u.

6 B. Stefanska, M. Arciemiuk, M. M. Bontemps-Gracz, M. Dzieduszycka, A. Kupiec, S. Martellib and E. Borowskia, Bioorg. Med. Chem., 2003, 11, 561-572.

7 S. Li, Y. Zhao, K. Wang, Y. Gao, J. Han, B. Cui and P. Gong, Bioorg. Med. Chem., 2013, 21, 2843-2855.

8 G. Zoidis, A. Sosic, S. Da Ros, B. Gatto, C. Sissi, F. Palluotto, A. Carotti and M. Catto, Bioorg. Med. Chem., 2017, 25, 26252634.

9 C.-K. Ryu and J. Y. Lee, Bioorg. Med. Chem. Lett., 2006, 16, 1850-1853.

10 M. P. Giovannoni, I. A. Schepetkin, L. Crocetti, G. Ciciani, A. Cilibrizzi, G. Guerrini, A. I. Khlebnikov, M. T. Quinn and C. Vergelli, J. Enzyme Inhib. Med. Chem., 2016, 31, 628639.

11 D. Masciocchi, A. Gelain, F. Porta, F. Meneghetti, A. Pedretti, G. Celentano, D. Barlocco, L. Legnani, L. Toma, B.-M. Kwon, A. Asaid and S. Villa, Med. Chem. Commun., 2013, 4, 1181.

12 C. Vernhet, E. Barbagallo, M. Rinaldi-Carmona and P. Roux, Fr. Demande, FR 2933697 A1 20100115, 2010.

13 E. Barbagallo, M. Rinaldi-Carmona, P. Roux and C. Vernhet, PCT Int. Appl., WO 2010004215 A2 20100114, 2010.

14 M. Alvarado, M. Barcelo, L. Carro, C. F. Masaguer and E. Ravina, Chem. Biodiversity, 2006, 3, 106-117.

15 Y. Sato, Y. Suzuki, K. Yamamoto, S. Kuroiwa and S. Maruyama, PCT Int. Appl., WO 2005121105 A1 20051222, 2005.

16 K. G. Pike and B. C. Barlaam, PCT Int. Appl., WO 2017162605 A1 20170928, 2017.

17 J. D. Lawson, M. Sabat, C. Smith, H. Wang, Y. K. Chen and T. Kanouni, Int. Appl., WO 2013148603 A1 20131003, 2013.

18 C. Alhambra, C. Becker, T. Blake, A. Chang, J. R. Damewood, T. Daniels, B. T. Dembofsky, D. A. Gurley, J. E. Hall, K. J. Herzog, C. L. Horchler, C. J. Ohnmacht, R. J. Schmiesing, A. Dudley, M. D. Ribadeneira, 
K. S. Knappenberger, C. Maciag, M. M. Stein, M. Chopra, X. F. Liu, E. P. Christian, J. L. Arriza and M. J. Chapdelaine, Bioorg. Med. Chem., 2011, 19, 2927-2938. 19 A. Goeminne, P. J. Scammells, S. M. Devine and B. L. Flynn, Tetrahedron Lett., 2010, 51, 6882-6885.

20 O. V. Vinogradova, V. N. Sorokoumov, S. F. Vasilevsky and I. A. Balova, Tetrahedron Lett., 2007, 48, 4907-4909.

21 O. V. Vinogradova, V. N. Sorokoumov, S. F. Vasilevskii and I. A. Balova, Russ. Chem. Bull. Int. Ed., 2008, 57, 1725-1733. 22 R. Dey and B. C. Ranu, Tetrahedron, 2011, 67, 8918-8924.

23 N. A. Al-Awadi, M. H. Elnagdi, Y. A. Ibrahim, K. Kaul and A. Kumar, Tetrahedron, 2001, 57, 1609-1614.

24 K. W. Woods, J. P. Fischer, A. Claiborne, T. Li, S. A. Thomas, G. D. Zhu, R. B. Diebold, X. Liu, Y. Shi, V. Klinghofer, E. K. Han, R. Guan, S. R. Magnone, E. F. Johnson, J. J. Bouska, A. M. Olson, R. de Jong, T. Oltersdorf, Y. Luo, S. H. Rosenberg, V. L. Giranda and Q. Li, Bioorg. Med. Chem., 2006, 14, 6832-6846.

25 I. S. Kim and P. H. Lee, Repub. Korea, KR 1740155 B1 20170525, 2017.

26 J. Yan, G. L. Tay, C. Neo, B. R. Lee and P. W. H. Chan, Org. Lett., 2015, 17, 4176-4179.

27 M. A. Berghot, E. M. Kandeel, A. H. Abdel-Rahman and A.-M. Marwa, Med. Chem., 2014, 4, 381-388.

28 A. D. T. Tuyet, L. Decuyper, T. P. Hoang, V. N. Doan, H. T. Nguyen, T. T. Nguyen, T. D. Huy, H. H. Nguyen, M. D'hooghe and T. V. Nguyen, Tetrahedron Lett., 2015, 56, 5855-5858.

29 T. J. Mason and D. Peters, Practical Sonochemistry, Ellis Horwood, New York, NY, USA, 1991.

30 J. L. Luche, Organic Sonochemistry, Plenum Press, New York, NY, USA, 1998.

31 T. J. Mason and J. P. Lorimer, Applied sonochemistry: uses of power ultrasound in chemistry and processing, Wiley-VCH, Weinheim, 2002.

32 A. Bazgir, S. Ahadi, R. Ghahremanzadeh, H. R. Khavasi and P. Mirzaei, Ultrason. Sonochem., 2010, 17, 447-452.

33 J. L. G. Ruano, A. Parra, L. Marzo, F. Yuste and V. M. Mastranzo, Tetrahedron, 2011, 67, 2905-2910.

34 P. Cintas, Ultrason. Sonochem., 2016, 28, 257-258.

35 B. Banerjee, Ultrason. Sonochem., 2017, 35, 1-14.

36 B. Banerjee, Ultrason. Sonochem., 2017, 35, 15-35.

37 G. Chatel, Ultrason. Sonochem., 2018, 40, 117-122.
38 S. Puri, B. Kaur, A. Parmar and H. Kumar, Curr. Org. Chem., 2013, 17, 1790-1828.

39 J. L. G. Ruano, A. Parra, L. Marzo, F. Yuste and V. M. Mastranzo, Tetrahedron, 2011, 67, 2905-2910.

40 M. B. Gawande, S. N. Shelke, R. Zboril and R. S. Varma, Acc. Chem. Res., 2014, 47, 1338-1348.

41 C. O. Kappe and A. Stadler, Microwave Theory. In Microwaves in Organic and Medicinal Chemistry, Wiley-Blackwell, 2005, pp. 9-28.

42 J. P. Tierney and P. Lidstrom, Microwave Assisted Organic Synthesis, Blackwell, Oxford, 2005.

43 A. Loupy, Microwaves in Organic Synthesis, Wiley-VCH, Weinheim, 2nd edn, 2006.

44 H. M. Al-Matar, S. M. Riyadha and M. H. Elnagdi, J. Heterocycl. Chem., 2007, 44, 603-607.

45 K. D. Khalil and H. M. Al-Matar, Molecules, 2012, 17, 1222512233.

46 K. M. Dawood and M. M. El-Deftar, Synthesis, 2010, 10301038.

47 H. A. Abdel-Aziz, H. S. A. El-Zahabi and K. M. Dawood, Eur. J. Med. Chem., 2010, 45, 2427-2432.

48 K. M. Dawood, A. M. Farag, M. M. El-Deftar, M. Gardiner and H. A. Abdelaziz, ARKIVOC, 2013, (iii), 210-226.

49 K. M. Dawood, M. B. Elamin and A. M. Farag, ARKIVOC, 2015, (vii), 50-62.

50 H. Behbehani, H. M. Ibrahim and K. M. Dawood, RSC Adv., 2015, 5, 25642-25649.

$51 \mathrm{H}$. Behbehani, K. M. Dawood, H. M. Ibrahim and N. S. Mostafa, Arabian J. Chem., 2017, DOI: 10.1016/ j.arabjc.2017.05.016, published online.

52 M. A. Al-Shiekh, H. Y. Medrassi, M. H. Elnagdi and E. A. Hafez, J. Chem. Res., 2007, 432-436.

53 H. M. E. Hassaneen and I. A. Abdelhamid, J. Heterocycl. Chem., 2017, 54, 1048-1053.

54 M. A. Al-Shiekh, H. Y. Medrassi, M. H. Elnagdi and E. A. Hafez, ARKIVOC, 2008, (xvii), 36-47.

55 F. Al-Qalaf, K. Almohammad, M. A. El-Apasery and H. Mahmoud, Eur. J. Chem., 2013, 4, 211-215.

56 The crystallographic data for compound 3a (ref. CCDC 1858311) can be obtained on request from the director, Cambridge Crystallographic Data Center, 12 Union Road, Cambridge CB2 1EW, UK.

57 C. O. Kappe, Angew. Chem., Int. Ed., 2004, 43, 6250-6284. 58 G. M. Sheldrick, Acta Crystallogr., 2008, A64, 112-122. 